\title{
Radon dans les habitations et cancers broncho-pulmonaires : mythe ou réalité?*
}

\author{
Marc DOUSSET **
}

\begin{abstract}
RÉSUMÉ
Ni les données dont nous disposons actuellement sur la mortalité par cancers broncho-pulmonaires en France à une époque où la consommation de tabac était beaucoup plus faible qu'aujourd'hui, ni celles qui, de nos jours, concernent les départements où les niveaux de radon dans les habitations sont les plus élevés ne justifient l'importance donnée actuellement à ce problème.
\end{abstract}

Les facteurs de risque proposés par la Publication CIPR 50 (1986) semblent être très nettement surestimés.

\section{ABSTRACT}

Neither the data presently available on mortality by broncho-pulmonary carcinomas in France at a time when tobacco consumption was much lower than nowadays, nor the present data relative to the "departements" where indoor radon levels are the highest justify the importance given now to the problem. The risk factors set forth in ICRP publication 50 (1986) seem very highly overestimated.

\section{INTRODUCTION}

Plusieurs faits ont contribué, depuis une dizaine d'années, à donner beaucoup d'importance au problème du radon dans les habitations:

1. Les résultats convergents des enquêtes épidémiologiques qui mettent en évidence une surmortalité par cancers broncho-pulmonaires (CBP) parmi les mineurs des mines souterraines d'uranium, de fer et d'autres métaux; cette surmortalité est attribuée au seul radon et extrapolée indûment au cas du radon dans les habitations;

- Communication présentée lors de la réunion SFRP "Exposition au radon dans les habitations: aspects techniques et sanitaires", Paris, 10-11 janvier 1989.

** Conseiller scientifique du SCPRI, ministère de la Santé. Adresse personnelle: 7, rue de la Gruerie, 91190 Gif-sur-Yvette. 
2. L'expérimentation animale sur le rat qui se révèle très sensible à l'action du radon; mais les études sur d'autres animaux de laboratoire, principalement sur le hamster, donnent des résultats souvent très différents;

3. La généralisation, dans tous les pays, de la mesure du radon dans les habitations qui a montré que, dans certains cas-limites, des personnes du public pouvaient inhaler dans leur appartement ou leur maison individuelle plus de descendants du radon que certains mineurs au cours de leur activité professionnelle;

4. Enfin, les modèles dosimétriques relatifs à l'arbre pulmonaire, fondés sur de nombreuses hypothèses, souvent contestables, et qui conduisent à des doses relativement élevées pour l'épithélium bronchique.

La Commission internationale de protection radiologique (CIPR) a résumé, en 1986 dans sa Publication 50 [4], les résultats de toutes ces spéculations en retenant principalement, un modèle dit "modèle du risque proportionnel" pour lequel l'action des différents toxiques cancérigènes, surtout celle du tabac, est multipliée par l'action du radon. Elle propose ainsi comme facteur de risque:

- pour les non-fumeurs (moyenne sur les deux sexes): une exposition chronique à $1 \mathrm{~Bq}$ (à l'eq.). $\mathrm{h} / \mathrm{m}^{3}$ chaque année entraînerait un risque pour la vie entière (risque-vie) de $6.10^{-9}$ (6 cas de décès par CBP par milliard de personnes) ou $4.10^{-3}$ pour une exposition de 1 WLM chaque année;

- - pour une population-type dont la composition (hommes - femmes et fumeurs - non fumeurs) est assez proche de la nôtre, cette même exposition chronique entraînerait un risque-vie de $2 \cdot 10^{-8}$ ( 2 cas de décès par CBP sur 100 millions de personnes), ou $1,3.10^{-2}$ pour une exposition de 1 WLM chaque année.

En fait, cette action du radon est très difficile à mettre en évidence, hormis le cas des mineurs. Si elle est réelle aux niveaux habituellement rencontrés dans les habitations, cette action est masquée par celle des multiples agents toxiques auxquels nous sommes exposés dans la vie quotidienne, principalement le tabac et les polluants de la vie urbaine ou industrielle. Aussi de nombreuses études portant sur des populations de régions à forte concentration en radon au Canada [5], en Finlande [1], en Suède [13], en Chine [3, 6, 11] ou aux Etats-Unis [2], ont-elles conduit à des résultats négatifs.

\section{OBJET DE L'ÉtUdE ET DONNÉES DE BASE}

L'objet de cette communication est de présenter les données actuellement disponibles sur la mortalité par CBP en France et d'en tirer quelques idées directrices sur l'importance du phénomène "radon dans les habitations". Cette étude s'appuie sur deux documents récents:

- les Causes de décès en France de 1925 à 1978 par deux chercheurs de l'Institut national d'études démographiques (INED) [12] ;

- I'Atlas de mortalité par cancer en France (1971-1978) publié en 1986 par I'INSERM [10]. 


\section{MORTALITÉ PAR CBP EN FRANCE DANS LES ANNÉES 1920-1930}

Malgré les difficultés qu'entraînent les révisions successives de la classification des maladies et le délicat problème de la répartition des décès de cause indéterminée, J. VALLIN et F. MESLE sont parvenus à décrire, avec une bonne cohérence, l'évolution dans le temps de la mortalité par CBP et à remonter ainsi jusqu'à l'année 1925. Le tableau I donne les taux de mortalité par CBP de 1925 à 1978; il s'agit de taux comparatifs, c'est-à-dire de taux rapportés à une même population dite "de référence"; celle-ci est la population française moyenne de 1978 (les deux sexes). On peut voir qu'en 1925 le taux (tous âges) de la mortalité par CBP était pour les femmes de l'ordre de 10 cas par million de personnes et par an $\left(10^{-5}\right)$; pour les hommes il était 3 fois plus élevé: $3 \cdot 10^{-5}$.

TABLEAU I

Evolution du taux comparatif * de décès par cancer broncho-pulmonaire de 1925 à 1978 en France

(Nombre de cas pour $10^{6}$ personnes et par an)

\begin{tabular}{|c|c|c|c|c|c|c|c|c|}
\hline Année & Hommes & Femmes & Année & Hommes & Femmes & Année & Hommes & Femmes \\
\hline 1925 & 29 & 10 & 1943 & 97 & 36 & 1961 & 343 & 51 \\
1926 & 32 & 12 & 1944 & 103 & 35 & 1962 & 362 & 53 \\
1927 & 36 & 14 & 1945 & 106 & 35 & 1963 & 387 & 56 \\
1928 & 36 & 15 & 1946 & 115 & 37 & 1964 & 402 & 60 \\
1929 & 39 & 15 & 1947 & 130 & 35 & 1965 & 423 & 59 \\
1930 & 40 & 15 & 1948 & 141 & 41 & 1966 & 446 & 59 \\
1931 & 43 & 18 & 1949 & 148 & 44 & 1967 & 452 & 63 \\
1932 & 45 & 17 & 1950 & 153 & 48 & 1968 & 476 & 61 \\
1933 & 45 & 17 & 1951 & 170 & 43 & 1969 & 495 & 60 \\
1934 & 47 & 17 & 1952 & 194 & 49 & 1970 & 489 & 58 \\
1935 & 48 & 19 & 1953 & 210 & 48 & 1971 & 519 & 60 \\
1936 & 48 & 21 & -1954 & 232 & 48 & 1972 & 546 & 61 \\
1937 & 51 & 23 & 1955 & 241 & 50 & 1973 & 555 & 62 \\
1938 & 54 & 22 & 1956 & 254 & 50 & 1974 & 577 & 62 \\
1939 & 53 & 20 & 1957 & 287 & 50 & 1975 & 591 & 61 \\
1940 & 68 & 26 & 1958 & 288 & 53 & 1976 & 630 & 61 \\
1941 & 72 & 29 & 1959 & 303 & 51 & 1977 & 649 & 62 \\
1942 & 76 & 31 & 1960 & 323 & 52 & 1978 & 684 & 65 \\
\hline
\end{tabular}

* Le taux comparatif a été calculé en prenant comme population de référence la population de la France (les deux sexes) en 1978, d'après [12].

La croissance de ces taux au cours de toutes les années qui ont suivi ainsi que l'augmentation du rapport entre les hommes et les femmes, de 3 à plus de 10 , sont généralement attribuées à l'usage du tabac et aux nuisances, de toute sorte, associées au développement, au cours de ces 63 années, de la vie urbaine et des activités industrielles. Pour les hommes la consommation de tabac est devenue relativement importante avec la guerre de 1914-1918 et le taux de mortalité par CBP en 1925 est en partie dû à 
l'action du tabac; mais il comprend également l'action des polluants liés aux activités professionnelles. Pour les femmes, on peut penser que la valeur de $10^{-5}$ est la plus proche du taux que l'on pourrait constater sans le tabac ni les autres polluants liés à la vie moderne. Cependant, il est sûr que cette valeur comprend un certain bruit de fond de cancers "spontanés" et de cancers dus aux agents toxiques dont la présence dans les habitations résulte des activités domestiques (gaz de combustion du bois, du charbon, etc.) sans compter l'effet indirect du tabagisme des hommes. Le taux de $10^{-5}$ doit donc être supérieur (sans qu'on puisse dire exactement de combien) à celui qui serait attribuable au seul radon chez les non-fumeurs. Pour s'exprimer en risque sur la vie comme le fait la CIPR 50, on peut obtenir un bon ordre de grandeur en multipliant le taux par 80 ans.

Si l'on attribue arbitrairement au seul radon le risque de $8.10^{-4}$ ainsi obtenu, on peut calculer la borne supérieure de l'action du radon chez les non-fumeurs.

A. RANNOU, à la suite des nombreux travaux dont il a rendu compte [7-9] estime à: $25 \mathrm{~Bq}$ (à l'éq.) $/ \mathrm{m}^{3}$ la valeur moyenne pour l'ensemble des habitations françaises de l'activité volumique du radon 222 (y compris l'équivalent du radon 220 souvent présent dans des proportions non négligeables).

En retenant la valeur classique de $6000 \mathrm{~h}$ par an pour le temps de séjour à l'intérieur des habitations et en négligeant l'inhalation de radon à l'extérieur, l'exposition moyenne s'établit à $175000 \mathrm{~Bq}$ (à l'éq.) . h/m $\mathrm{m}^{3}$ (ou 0,28 WLM) par an, ce qui conduit à une limite supérieure du risque-vie attribuable au radon pour les non-fumeurs de $8.10^{-4} / 1,75.10^{5} \simeq 4,6.10^{-9}$ pour une exposition chronique à $1 \mathrm{~Bq} \cdot \mathrm{h} / \mathrm{m}^{3}$ par an ou encore: $2,9.10^{-3}$ pour $1 \mathrm{WLM}$ par an. Les valeurs avancées par la CIPR 50 (à savoir: $6.10^{-9}$ ou $4.10^{-3}$ ) sont supérieures à ces bornes, ce qui conduit à penser qu'elles sont nettement surestimées.

On peut évidemment reprocher au raisonnement précédent de s'appuyer sur une valeur contestable du taux de mortalité par CBP; dans les années 1920-1930, d'autres causes de mortalité, en particulier les maladies infectieuses, ont pu masquer, dans chaque classe d'âge, nombre de décès par CBP et, d'autre part, la qualité des diagnostics n'était certainement pas ce qu'elle est aujourd'hui. Cependant, une autre observation, qui échappe à ces critiques car elle porte sur la mortalité par CBP chez les femmes à une période beaucoup plus récente, corrobore la conclusion précédente.

Si l'on considère cette mortalité féminine pour les trois années 1968 , 1969 et 1970 dans les départements du Finistère, du Morbihan et de la Haute-Vienne dans lesquels les mesures montrent une activité volumique moyenne du radon dans les habitations qui est au moins le double de la moyenne générale pour la France, le taux comparatif de mortalité par CBP pour l'ensemble de ces trois départements $\left(5,6.10^{-5}\right)$ est inférieur à celui de la France entière $\left(6.10^{-5}\right)$. Le facteur de risque (non-fumeur) de la CIPR 50 , $4.10^{-3} /$ WLM chaque année, aurait conduit à un taux supérieur à $7.10^{-5}$; 
l'effet que peut avoir un doublement de la concentration en radon est largement contrebalancé par celui de toutes les autres variables économiques, sociales et autres.

\section{MORTALITÉ PAR CBP DANS LES DÉPARTEMENTS FRANÇAIS ENTRE 1971 ET 1978}

Pour les hommes, avec la proportion de fumeurs qu'on trouve dans les années 1970-1980, on dispose des données de l'Atlas de mortalité par cancer publié par A. REZVANI et coll. [10] en 1986. Le rapport de mortalité normalisé (SMR) pour les CBP, pour l'ensemble des années allant de 1971 à 1978, y est calculé pour chaque département français. Ces valeurs sont portées en ordonnées sur la figure 1. La population masculine de l'ensemble de la France ayant été choisie comme population de référence, il est logique que les valeurs trouvées varient de valeurs inférieures à $1(0,5$ environ au minimum) à des valeurs supérieures à 1 (1,6 maximum) selon l'importance de la mortalité par CBP dans chaque département. En abscisses est portée, non pas la consommation de tabac, mais la vente de tabac par la SEITA (statistiques 1968) exprimée en grammes par habitant pour chaque département. En l'absence de données précises sur les consommations de tabac, ces ventes par habitant constituent pour les hommes un bon indicateur.

On peut constater que la majeure partie des points représentatifs des départements (83 sur 94) sont concentrés dans un nuage ascendant qui exprime bien la corrélation entre la vente de tabac et la mortalité par CBP. Le coefficient de corrélation qui s'établit aux environs de 0,8 est hautement significatif. Au-dessous de ce nuage se trouvent 4 points qui paraissent aberrants. Pour la Lozère, où pourtant les sols granitiques font présager des concentrations élevées en radon, il est difficile de trouver une explication; mais pour les Alpes maritimes, il est logique de penser que le caractère très touristique de ce département implique d'importants achats de tabac par des personnes dont le domicile est hors du département. Pour les Alpes de Haute-Provence et les Hautes-Alpes, la frontière italienne et les achats de contrebande peuvent, peut-être, en partie expliquer leur position excentrique sur la figure. Au-dessus du nuage se trouvent 6 à 7 points qui correspondent à des départements à forte concentration industrielle. Au sein du nuage se trouvent 9 des 11 départements dans lesquels on sait que la concentration moyenne en radon dans les habitations est de 2 à 3 fois celle de l'ensemble de la France. Ils s'insèrent à leur place au même niveau du SMR que les autres départements à vente de tabac égale. La Loire, avec son important bassin industriel stéphanois est le seul à être au-dessus du nuage et à être aligné avec les principaux départements à grande concentration industrielle.

Comparée à l'action du tabac et à celle des conditions de vie auprès des grands bassins miniers et industriels, l'action du radon dans les habitations paraît donc de peu d'importance pour les hommes, même fumeurs.

Pour obtenir une information plus quantitative nous avons comparé 2 groupes de départements pour lesquels les ventes de tabac par habitant 
站:

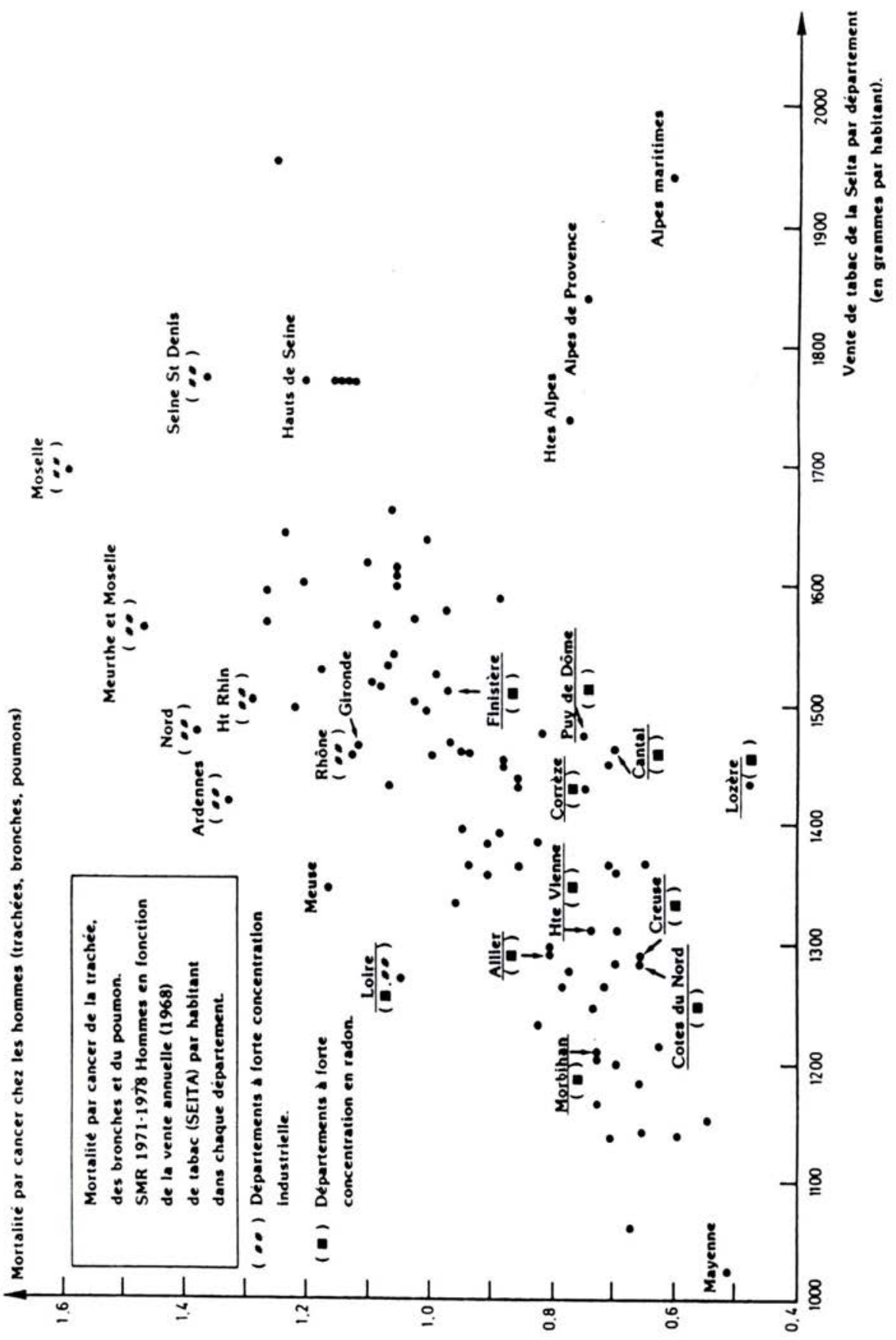


sont pratiquement les mêmes, environ $1300 \mathrm{~g} / \mathrm{an}$, et qui se situent tous dans un contexte à dominance agricole. Le premier groupe (Lot, Lot-etGaronne, Gers et Manche) présente une concentration en radon dans les habitations qui est environ la moitié de celle que l'on trouve dans le second groupe (Allier, Côtes-du-Nord, Creuse et Haute-Vienne).

$\mathrm{Si}$ on prend le groupe de départements où il y a le moins de radon comme témoin et si on calcule le nombre de décès par CBP que l'on devrait dénombrer dans le second groupe, en tenant compte des différences de populations et de leur distribution en classes d'âge, on trouve que, pour les 8 années de 1971 à 1978, le nombre attendu serait 2506; en fait, on en observe 2369 . La différence (-137) est donc négative et significative avec $\mathrm{p}=0,003$. L'application du facteur de risque de la CIPR $\left(2 \cdot 10^{-8} \mathrm{par} \mathrm{Bq} \cdot \mathrm{h} / \mathrm{m}^{3}\right)$ conduirait à +279 cas.

Un tel écart, après avoir exclu le tabac et la proximité des grandes concentrations industrielles, incite à rejeter le modèle du risque proportionnel et la valeur du facteur de risque proposé par la CIPR pour les expositions chroniques au radon comme celles que l'on rencontre dans les conditions habituelles de la vie domestique. Le risque est certainement très faible et l'importance que l'on tend à donner actuellement au radon dans les habitations est démesurée. II y a, en hygiène publique, des problèmes autrement plus sérieux auxquels il faudrait consacrer des ressources qui risquent, si la psychose du radon continue à se développer, d'être affectées à lutter contre un danger quelque peu mythique.

\section{RÉFÉRENCES}

[1] CASTREN O. et al. - Radon measurements in Finish houses. In: Indoor exposure to natural radiation and associated risk assessment, Anacapri, Oct. 3-5, 1983. Radiat. Prot. Dos., 1984, 7 (1/4) 333-336.

[2] COHEN B.L. - A national survey of radon in homes and correlating factors. Health Phys., 1986, 51 (2) 175-183 et Health Phys., 1987, 52 (5) 629-636.

[3] HOFMANN W., KATZ R. CHUXIANG Z. - Lung cancer risk at low doses of alpha particles. Health Phys., 1986, 51 (4) 457-468.

[4] INTERNATIONAL COMMISSION ON RADIOLOGICAL PROTECTION (ICRP). Lung cancer risk from indoor exposures to radon daughters, (ICRP Publication 50). Oxford: Pergamon press 1986.

[5] LETOURNEAU E. et al. - Lung cancer mortality and indoor radon concentration in 18 Canadian cities. Health physics society 16 th midyear topical meeting, In: Epidemioligy applied to health physics, Albuquerque, Jan. 9-13, 1983. CONF 830101.

[6] LUXIN W. - Health survey in high background radiation areas in China. Science, 1980, 209 (4459) 877-880.

[7] RANNOU A. - Contribution à l-étude du risque lié à la présence du radon 220 et du radon 222 dans l'atmosphère des habitations. Rapport CEA - R - 5378, 1987. 
[8] RANNOU A. - Indoor radon exposure assessment: an overview of the french survey. In: Indoor radon II, 2nd APCA international specialty conference, Cherry Hill, April 6-10, 1987. Cherry Hill: APCA, 1987, 78-91.

[9] RANNOU A. et TYMEN G. - Les résultats des campagnes de mesures et facteurs explicatifs associés. In: Exposition au radon dans les habitations: aspects techniques et sanitaires, Paris, 10-11 janv. 1989. Fontenay-aux-Roses: SFRP, 1989, 41-63 et Radioprotection, 1989, 24 (4) 301-319.

[10] REZVANI A., DOYON F., FLAMANT R. - Atlas de la mortalité par cancer en France. Statistiques de santé. Paris: Éditions INSERM, 1986.

[11] SHAOJIAN Z. - Survey of mortality from malignant tumors in high background area of Guangdong. Chin. J. Radiol. Med. Prot. 1982, 2 (2) p. 48.

[12] VALLIN J. et MESLÉ F. - Les causes de décès en France de 1925 à 1978 (Institut national d'études démographiques, travaux et documents, annexe $X$ du cahier 115). Paris: Presses Universitaires de France, 1988.

[13] WILANDER G., 1986. - Personal communication to B.L. Cohen. Health Phys., 1987, 52 (5) 629-636. 